Review/Meta-analyses

\title{
Long-term clinical and functional course of borderline personality disorder: A meta-analysis of prospective studies
}

\author{
Irene Álvarez-Tomás ${ }^{\mathrm{a}, \mathrm{b}, *}$, José Ruiz ${ }^{\mathrm{a}}$, Georgina Guilera ${ }^{\mathrm{c}, \mathrm{d}}$, Arturo Bados $^{\mathrm{a}}$ \\ a Department of Clinical Psychology and Psychobiology, Division of Personality, Assessment and Psychological Treatments, Faculty of Psychology, University of \\ Barcelona, Spain \\ b CSMA Alt Penedès, Hospital Sagrat Cor Martorell, Sisters Hospitallers, Barcelona, Spain \\ ${ }^{\mathrm{c}}$ Department of Social Psychology and Quantitative Psychology, Faculty of Psychology, University of Barcelona, Spain \\ d Institute of Neurosciences, University of Barcelona, Spain
}

\section{A R T I C L E I N F O}

\section{Article history:}

Received 17 July 2018

Received in revised form 24 October 2018

Accepted 25 October 2018

Available online 24 December 2018

\section{Keywords:}

Meta-analysis

Borderline personality disorder

Long-term

Course

Follow-up

Prognosis

Therapy outcome

\begin{abstract}
A B S T R A C T
Background: This meta-analytic review is the first to synthesise findings from prospective research on the long-term course of borderline personality disorder in adult clinical populations.

Methods: Systematic searches were conducted in Medline, PsycINFO, PsycArticles, PubMed and Scopus within the period 1990-2017. Inclusion criteria were: (1) adult BPD sample diagnosed by a validated, semi-structured interview; (2) at least two prospective assessments of outcomes; and (3) follow-up period $\geq 5$ years. Quality of evidence was rated with the Systematic Assessment of Quality in Observational Research (SAQOR). Four outcomes were meta-analysed using mixed-effect methods: remission from BPD diagnosis, completed suicide, depressive symptoms, and functioning. Potential moderators regarding the natural course and the initial treatment received were studied.

Results: Eleven studies met the inclusion criteria, with 837 participants from nine countries being followed. Between $50 \%$ and $70 \%$ of the BPD patients achieved remission in the long-term. Significant reductions in depression and functional impairment were also found. Mean suicide rate ranged from $2 \%$ to $5 \%$. Younger age was associated with higher likelihood for remission. Being female was correlated with lower functional improvement. Despite some positive trends, there were no significant associations between treatment moderators and the long-term outcome.

Conclusions: Findings suggest that the course of BPD is characterised by symptomatic amelioration and a slight functional improvement in the long-term. Age and gender modulate the long-term prognosis and should be considered to adapt treatment resources. Further research is required to draw robust conclusions on the long-term effects of psychotherapeutic interventions.
\end{abstract}

(c) 2018 Elsevier Masson SAS. All rights reserved.

\section{Introduction}

Over the last few decades, there has been growing evidence that the natural course of borderline personality disorder (BPD) is characterised by its plasticity, with consecutive periods of remission and relapse, and shows a trend towards symptomatic amelioration over time. Despite that, adults with BPD appear to frequently suffer from poor psychosocial functioning in the long-term [1,2,3]. Bearing this in mind, Paris suggested that treatment efficacy should be assessed in terms of their contribution to enhance the natural

\footnotetext{
* Corresponding author at: Department of Clinical Psychology and Psychobiology, Division of Personality, Assessment and Psychological Treatments, Faculty of Psychology, University of Barcelona, Spain.

E-mail address: irene.alvareztomas@gmail.com (I. Álvarez-Tomás).
}

process of the disorder. In this respect, it is noteworthy to study the impact of psychotherapeutic interventions in patients with BPD in the long-term, both in the symptomatic and functional domains $[2,3]$.

Two main prospective studies provided evidence on a wide range of aspects related to the long-term course of adults with BPD: the McLean Study of Adult Development (MSAD) and the Collaborative Longitudinal Personality Study (CLPS) $[4,5,6]$. Both research projects demonstrated that it is common for BPD patients to experience periods of symptomatic remission over time, reporting high cumulative rates by 10 years ( $85 \%$ 12-month remission in CLPS; 93\% 2-year remission in MSAD) [6]. At 16 years, the MSAD also reported that $78 \%$ of BPD patients had achieved a long-lasting remission of eight consecutive years [7]. Nevertheless, the recovery of psychosocial functioning was less consistent than symptomatic remission, oscillating between a steady functional 
impairment reported by the CLPS and a slight improvement by the MSAD [6]. Besides, patients with BPD were mostly not able to achieve normal levels of functioning in the long-term: only 33\% had good functioning after 6 years in MSAD; and just 21\% did so after 10 years in CLPS $[8,9]$.

Apart from the longitudinal research conducted in the US population, other naturalistic studies were carried out in different countries (i.e., Spain, Canada, Finland, and Germany) providing prospective data for a period of five years or more. Findings in these studies add to the evidence that the course of BPD is characterised by symptomatic improvement, although remission rates ranged widely from $31 \%$ to $81 \%$, which were informed at different time points of follow-up [10,11,12,13]. Álvarez-Tomás et al. also reported a slight improvement of psychosocial functioning in a Spanish sample followed up at 10 years, although dysfunctional levels of adjustment were maintained [10]. Moreover, only one third of subjects with BPD, on average, achieved both symptomatic and functional recovery at a 14-year follow-up in a German study [13].

Further analysis of potential moderators should be required to take into account variations on the course of BPD among studies. In this regard, previous longitudinal research pointed out the following factors as predictors of long-term outcomes: demographic characteristics, childhood experiences, stressful life events, treatment history, psychopathologic comorbidity, personality traits, and premorbid psychosocial functioning [14,15,16,17,18].

In addition to naturalistic research, clinical trials of psychotherapeutic interventions for BPD have recently shown greater interest in their long-term outcomes, reporting follow-up data for five years or more $[19,20,21,22,23]$. This longitudinal perspective on efficacy studies underlines the relevance of treatment as a potential factor of change over time. Nevertheless, findings are controversial among studies with respect to a differential impact of specialised therapies in comparison to treatment as usual (TAU) on diagnostic change and social functioning in the long-term. Bateman and Fonagy reported significant differences on remission favouring mentalisation-based treatment (MBT) over TAU ( $86 \%$ vs. $13 \%$ ), but this effect was not found for other specialised therapies $[19,20,21]$. Antonsen et al. reported better outcomes for the social functioning of a specialised therapy for personality disorders compared to TAU, in contrast to findings in the Boscot trial indicating a similar impact of treatment interventions [19,21]. As far as we are aware, there are no published meta-analyses that synthesise current evidence on the long-term outcome of treatment interventions in BPD or that combine these findings with those from naturalistic prospective research. This may contribute to the study of long-term treatment effects in the context of the natural course of the disorder.

Thus, our objective was to conduct a meta-analysis of studies reporting the prospective results on the long-term course of BPD, considering both naturalistic and post-treatment follow-up research. In case of heterogeneity among studies, we were interested to study the effect of potential moderators related to the natural course (e.g., age, gender, time of follow-up, psychiatric comorbidity, and initial level of functioning) and those related to the treatment interventions received. The following questions were addressed: (1) What characterises the long-term course of BPD in adulthood, both in clinical and functional domains? (2) Are there significant moderators that influence the long-term course of the disorder?

\section{Materials and methods}

\subsection{Search strategy}

Our literature review was guided by the PRISMA standards for systematic reviews [24]. Bibliographic searches were conducted in
Medline, PsycINFO, PsycArticles, PubMed, and Scopus to identify relevant literature during the period 1990-2017. The searching strategy was a combination of the following two steps: (1) either "borderline personality disorder" or "personality disorders" were used in addition to terms indicating a temporal dimension, i.e., "follow-up", “course", "longitudinal", "long-term", or "maintenance", in the title or abstract field; (2) the terms "borderline personality disorder" and either "treatment*", "therap*", "psychotherap", "intervention*", or "program*" were combined in the title field and added to similar temporal terms in the abstract field to identify follow-up clinical studies in BPD samples. We examined the references of all included articles to identify other relevant publications and contacted authors to obtain additional information. Dissertations and conference papers were also reviewed.

\subsection{Selection criteria}

Inclusion criteria were: (1) the presence of an adult BPD sample or subsample in the study, diagnosed by a validated, semistructured interview; (2) at least two assessments with repeated outcome measures related to the course of BPD; and (3) a followup period of 5 years or more.

\subsection{Data collection}

Data extraction of the selected studies was independently completed by two investigators using an agreed coding protocol (available upon request). The authors of three studies were contacted to request additional information. A response was obtained from a study pending publication, which was included in the meta-analyses. The level of agreement between the coders was high (average agreement percentage $=95.53$; average Kappa $=.91$ ).

\subsection{Outcome variables}

The comparable measures reported by at least three studies were considered as the minimum measures needed to successfully perform a meta-analysis, finally resulting in four outcomes of interest: remission, completed suicide, depression, and functioning.

As a measure of remission, the percentage of subjects who achieved diagnostic remission for BPD at a specific follow-up point was used; this measure differs from the cumulative remission rate, which is defined as the percentage of subjects who achieved a particular period of remission throughout the duration of the follow-up. Completed suicide was computed as the number of subjects who completed suicide during follow-up divided by the total number of subjects included at baseline in the respective study.

Depression and functioning were studied through the means of diverse instruments used in the studies. Their comparability was determined by consensus of the authors.

\subsection{Definition of moderators}

The potential factors reported by at least three studies were considered as moderators; age at baseline, gender, time of followup, comorbidity with mood disorders at baseline, initial level of functioning, presence and type of controlled treatment and its length in months, and total hours of therapy were studied.

As a measure of the initial level of functioning, we converted the mean scores of the functioning scales at baseline into a z-score, comparing the mean values in each study with those reported for these instruments in clinical populations from the corresponding countries, which mainly consisted of outpatients with anxiety or affective disorders $[25,26,27,28,29,30]$. 
Regarding treatment, we analysed the impact of receiving a controlled treatment at the outset of the follow-up, comparing samples of naturalistic studies with those of follow-up clinical trials. Any treatment received in experimental conditions (e.g., predetermined prescription or length of treatment, attendance monitoring, etc.) was considered as a controlled treatment. Secondly, we compared those subgroups receiving specialised therapy for BPD or other personality disorders with those receiving TAU in follow-up clinical studies. Finally, we studied the length of the controlled treatment in these subgroups and, where possible, the total hours of formal therapy received, including both individual and group modalities. To this end, we calculated the total amount of hours of formal therapy, multiplying the number of therapy sessions by the hours per session.

\subsection{Quality assessment}

A quality assessment of studies was performed with the SAQOR, which was developed to assess quality in psychiatric research [31]. We followed the adapted guidelines used to assess both intervention and longitudinal studies in a former meta-analysis [32]. According to the purpose of the present review, quality was assessed considering the presence of BPD diagnosis as the exposure variable, regardless of the original design or aims of the studies. Gender/age, psychiatric comorbidity, type of initial treatment, amount of therapy during the follow-up, and other variables were considered among factors that might affect the association between BPD diagnosis and the long-term outcome. The control group domain was not applied, since the results of these samples were not analysed. The final SAQOR ratings were modified to maintain a similar sensitivity of the scale (see Table 1). Two of the authors completed the ratings, with an $89 \%$ average interrater agreement in domains' scores (average Kappa = .78) and an $82 \%$ in final ratings (Kappa $=.63$ ).

\subsection{Meta-analyses}

\subsubsection{Effect size computation}

For remission and completed suicide, the event rate itself was treated as a measure of effect size; event rates were converted into percentages. In the study of depression and functioning, the effect size used was Hedges' unbiased $g$ standardised mean difference and was calculated by subtracting the mean scores at baseline from the mean at the follow-up, divided by the standard deviation within groups [33]. Since test-retest information is used to adjust the standard errors of the effect size estimates, we used test-retest correlation coefficients of $0.50, .60$ and, .70 (presented here are those results based on a correlation of .50; the remaining analyses may be obtained upon request, but no substantial differences were found). Hedges' $g$ was computed in such a way that positive values indicate more of a particular outcome (i.e., more depression and more functioning).

\subsubsection{Meta-analytic procedures}

We ran four separate meta-analyses, one for each outcome variable of interest (i.e., remission, completed suicide, depression, and functioning). The likelihood of publication bias was tested using the Egger's regression test [34] in those meta-analyses with at least ten of the studies (i.e., remission and suicide) and the trimand-fill procedure [35] in the remaining number [36].

To determine whether each set of independent estimates shared a common effect size, we computed the homogeneity $Q$ statistic and the $I^{2}$ index (i.e., the estimated percentage of the total amount of variability that can be attributed to heterogeneity) $[37,38]$. Since heterogeneity was observed in three of the four meta-analyses, effect sizes were combined under the random- effects model using the restricted maximum-likelihood estimation to estimate the amount of heterogeneity [39]. We obtained an estimate of the overall effect size (i.e., event rate or Hedges' $g$ ) for each outcome of interest, which was also tested by computing a $95 \%$ confidence interval $(\mathrm{CI})$ and the associated $p$ value.

To examine the relationship between the moderators and the effect sizes, a mixed-effects model was used (i.e., random-effects model with moderators), using restricted maximum-likelihood to estimate residual heterogeneity. For quantitative moderators, we report the estimated parameter, its $95 \% \mathrm{CI}$, the $Q_{M}$ (i.e., heterogeneity accounted for by the moderator), and the associated $p$ value. For categorical variables, we report the estimated effect sizes within each level of a moderator with the corresponding $95 \%$ $\mathrm{CI}$, and the $Q_{B}$ and its statistical significance. Analyses were conducted with the Comprehensive Meta-Analysis (CMA) software [40].

\section{Results}

\subsection{Description of studies}

A total of 1718 records were identified through database searching and other sources. Fig. 1 describes the flow chart of the selection process. After removing duplicates, 769 records were screened that fulfilled inclusion criteria. Reasons for exclusion were: the design of the study was not prospective, the absence of a specified BPD sample, the lack of repeated outcome measures, and a follow-up period that did not course entirely through adulthood or was shorter than five years. Finally, twenty full-text articles corresponding to eleven prospective studies were selected and included in at least a meta-analysis.

Table 1 describes the studies included in the meta-analyses and the outcome variables which were analysed from each of them. There were five clinical trials with long-term posttreatment follow-ups and six naturalistic studies without a controlled treatment phase. The methodological qualities of the studies are displayed in Table 1 . The time from baseline to follow-up ranged from five to fourteen years, with a median value of six years. Overall, 837 participants from nine countries completed both assessments. The majority of studies showed a retention rate of more than 60 percent, with the exception of the naturalistic study with a fourteen-year follow-up [13]. Mean ages at baseline oscillated between 27 and 35 years, and the percentages of women were between $47 \%$ and $100 \%$. Percentages of comorbidity with mood disorders at baseline fluctuated between $37.7 \%$ and $100 \%$; this last percentage was due to one study conducted in a sample of primary care patients diagnosed with both major depressive disorder and BPD [12]. Recruitment settings in the rest of studies were outpatient or inpatient psychiatric services.

\subsubsection{Measures of outcome}

Selected studies reported follow-up data in several areas, i.e., the presence of BPD diagnosis and BPD symptomatic domains, dimensional personality traits, Axis II and Axis I comorbidity, general psychopathology, depression, anxiety, interpersonal problems, suicidal behaviour, psychosocial and global functioning, and quality of life. We only found comparable data for the four outcomes analysed (see Table 1).

Regarding remission, the CLPS was excluded from the metaanalysis due to its reporting of cumulative remission rates [9]. Aside from this type of measure, the MSAD also reported a specific remission rate at a six-year follow-up that was considered in the analyses [49]. The presence/absence of BPD diagnosis was generally assessed by similar instruments at baseline and follow-up, with the exception of two studies $[13,20]$. 
Table 1

Description of studies included in the meta-analyses $(N=837)$.

\begin{tabular}{|c|c|c|c|c|c|c|c|c|c|c|c|}
\hline $\begin{array}{l}\text { Study } \\
\text { Authors, year }\end{array}$ & Country & Quality $^{\mathrm{a}}$ & $\begin{array}{l}\text { Inclusion } \\
\text { Criteria } \\
\text { (measure) }\end{array}$ & $\begin{array}{l}\text { Years } \\
\text { FU } \\
\text { (\% Re- } \\
\text { tention) }\end{array}$ & $\begin{array}{l}\text { Presence/Type } \\
\text { Controlled } \\
\text { Treatment }\end{array}$ & $\begin{array}{l}\text { Initial } \\
N \\
(N \text { at } \\
\text { FU) }\end{array}$ & $\begin{array}{l}\text { Mean } \\
\text { Age } \\
(\% \\
\text { Women) }\end{array}$ & $\begin{array}{l}\% \text { Mood } \\
\text { disorder }\end{array}$ & $\begin{array}{l}\text { Completed } \\
\text { Suicide } \\
N(\%)\end{array}$ & $\begin{array}{l}\text { Remission } \\
\text { Rate } \\
\text { (measure) }\end{array}$ & $\begin{array}{l}\text { Depression/ } \\
\text { Functioning } \\
\text { measures }\end{array}$ \\
\hline $\begin{array}{l}\text { (1) Conversational } \\
\text { Therapy Trial } \\
\text { Stevenson et al., 1992, } \\
2005 \\
{[23,41]^{\mathrm{b}}}\end{array}$ & Australia & Moderate & $\begin{array}{l}\text { DSM-III } \\
\text { (DIB) }\end{array}$ & $\begin{array}{l}6 y \\
(63)\end{array}$ & $\begin{array}{l}\text { CT/ Specialised } \\
\text { therapy }\end{array}$ & $\begin{array}{l}48 \\
(30)\end{array}$ & $\begin{array}{l}29.4 \\
(63.3)\end{array}$ & - & $0(0)$ & $\begin{array}{l}40 \\
(\mathrm{DIB})\end{array}$ & - \\
\hline $\begin{array}{l}\text { (2) MBT Trial } \\
\text { Bateman \& Fonagy, } \\
\text { 1999, 2001, 2008 } \\
{[20,42,43]}\end{array}$ & UK & Moderate & $\begin{array}{l}\text { DSM-III-R } \\
\text { (SCID/DIB) }\end{array}$ & $\begin{array}{l}8 y \\
(93)\end{array}$ & $\begin{array}{l}\text { CT/ Specialised } \\
\text { therapy } \\
\text { CT/TAU }\end{array}$ & $\begin{array}{l}22 \\
(22) \\
22 \\
(19)^{\mathrm{c}}\end{array}$ & $\begin{array}{l}30.3 \\
(68) \\
33.3 \\
(47)\end{array}$ & 62 & $\begin{array}{l}0(0) \\
1(4.5)\end{array}$ & $\begin{array}{l}86 \\
13 \\
\text { (ZAN-BPD) }\end{array}$ & - \\
\hline $\begin{array}{l}\text { (3) Boscot Trial } \\
\text { Davidson et al., 2006, } \\
2010 \\
\text { Davidson, Norrie et al., } \\
2006 \\
\text { Palmer et al., } 2006 \\
\text { [21,44,45,46] }\end{array}$ & UK & High & $\begin{array}{l}\text { DSM-IV } \\
\text { (SCID-II) }\end{array}$ & $\begin{array}{l}6 y \\
(72)\end{array}$ & $\begin{array}{l}\text { CT/ Specialised } \\
\text { therapy } \\
\text { CT/ TAU }\end{array}$ & $\begin{array}{l}54 \\
(43) \\
52 \\
(33)\end{array}$ & $\begin{array}{l}32.4 \\
(83.3) \\
31.4 \\
(84.6)\end{array}$ & $\begin{array}{l}- \\
-\end{array}$ & $\begin{array}{l}1(1.9) \\
1(1.9)\end{array}$ & $\begin{array}{l}56 \\
52 \\
(\text { SCID-II) }\end{array}$ & $\begin{array}{l}\text { BDI-II/ } \\
\text { SFQ }\end{array}$ \\
\hline $\begin{array}{l}\text { (4) Ullevål Trial } \\
\text { Antonsen et al., } 2017 \\
\text { Arnevik et al., } 2010 \\
{[19,47]}\end{array}$ & Norway & Moderate & $\begin{array}{l}\text { DSM-IV } \\
\text { (SCID-II) }\end{array}$ & $\begin{array}{l}6 y \\
(65)\end{array}$ & $\begin{array}{l}\text { CT/ Specialised } \\
\text { therapy } \\
\text { CT/ TAU }\end{array}$ & $\begin{array}{l}27 \\
(19)^{d} \\
25 \\
(15)^{d}\end{array}$ & $\begin{array}{l}29 \\
(85)\end{array}$ & 88 & $\begin{array}{l}1(3.7) \\
0(0)\end{array}$ & $\begin{array}{l}90 \\
93 \\
(\mathrm{SCID}-\mathrm{II})\end{array}$ & $\begin{array}{l}\text { BDI/ } \\
\text { GAF } \\
\text { WSAS }\end{array}$ \\
\hline \multirow[t]{2}{*}{$\begin{array}{l}\text { (5) SKIP Trial } \\
\text { Sahin et al., } 2017 \text { [22] }\end{array}$} & Sweden & Moderate & $\begin{array}{l}\text { DSM-IV } \\
\text { ICD-10 } \\
(\text { DIP-I })\end{array}$ & $5 y$ & $\begin{array}{l}\text { CT/ Specialised } \\
\text { therapy } 1\end{array}$ & $\begin{array}{l}36 \\
(35)^{\mathrm{e}}\end{array}$ & $\begin{array}{l}31 \\
(100)\end{array}$ & 37.7 & - & - & GAF \\
\hline & & & & & $\begin{array}{l}\text { CT/ Specialised } \\
\text { therapy } 2 \\
\text { CT/ TAU }\end{array}$ & $\begin{array}{l}35 \\
(32)^{\mathrm{e}} \\
35 \\
(29)^{\mathrm{e}}\end{array}$ & $\begin{array}{l}30.6 \\
(100) \\
27.9 \\
(100)\end{array}$ & & & & \\
\hline $\begin{array}{l}\text { (6) McMaster Study } \\
\text { Links et al., 1995, } 1998 \\
{[11,48]}\end{array}$ & Canada & Moderate & $\begin{array}{l}\text { DSM-III } \\
(\mathrm{DIB} \geq 7)\end{array}$ & $\begin{array}{l}7 y \\
(65)\end{array}$ & $\begin{array}{l}\text { No controlled } \\
\text { treatment }\end{array}$ & $\begin{array}{l}88 \\
(57)\end{array}$ & $\begin{array}{l}34.7 \\
(93)\end{array}$ & 93 & - & $\begin{array}{l}52.6 \\
(\mathrm{DIB}<7)\end{array}$ & - \\
\hline $\begin{array}{l}\text { (7) MSAD Study } \\
\text { Zanarini et al., } 2003 \\
\text { [49] }\end{array}$ & US & High & $\begin{array}{l}\text { DSM-III-R } \\
\text { (DIB-R } \geq 8 \\
\text { /DIPD-R) }\end{array}$ & $\begin{array}{l}6 y \\
(91)\end{array}$ & $\begin{array}{l}\text { No controlled } \\
\text { treatment }\end{array}$ & $\begin{array}{l}290 \\
(264)\end{array}$ & $\begin{array}{l}26.9 \\
(80.3)\end{array}$ & 96.9 & $11(3.8)$ & $\begin{array}{l}68.6 \\
(\mathrm{DIB}-\mathrm{R}<8 \\
\text { /DIPD-R) }\end{array}$ & $\mathrm{GAF}^{\mathrm{f}}$ \\
\hline $\begin{array}{l}\text { (8) CLPS Study } \\
\text { Skodol et al. 2005, } \\
\text { Gunderson et al., } 2011 \\
{[9,50]}\end{array}$ & US & High & $\begin{array}{l}\text { DSM-IV } \\
\text { (DIPD-IV) }\end{array}$ & $\begin{array}{l}10 y \\
(63)\end{array}$ & $\begin{array}{l}\text { No controlled } \\
\text { Treatment }\end{array}$ & $\begin{array}{l}175 \\
(111)\end{array}$ & $\begin{array}{l}32.1 \\
(75)\end{array}$ & 70.9 & $1(0.6)$ & - & $\mathrm{GAF}^{\mathrm{f}}$ \\
\hline $\begin{array}{l}\text { (9) Vaanta Primary Care } \\
\text { Depression Study } \\
\text { Riihimäki et al., } 2014 \\
\text { [12] }\end{array}$ & Finland & Moderate & $\begin{array}{l}\text { DSM-IV } \\
\text { (SCID-II) }\end{array}$ & $\begin{array}{l}5 y \\
(83)\end{array}$ & $\begin{array}{l}\text { No controlled } \\
\text { treatment }\end{array}$ & $\begin{array}{l}35 \\
(29)\end{array}$ & $\begin{array}{l}32 \\
(86)\end{array}$ & 100 & - & $\begin{array}{l}31 \\
(\mathrm{SCID}-\mathrm{II})\end{array}$ & $\begin{array}{l}\text { 17-HDRS } \\
\text { BDI/ } \\
\text { SOFAS }\end{array}$ \\
\hline $\begin{array}{l}\text { (10) Alvarez-Tomás et al., } \\
2017 \text { [10] }\end{array}$ & Spain & Moderate & $\begin{array}{l}\text { DSM-IV } \\
(\mathrm{DIB}-\mathrm{R} \geq 6 \\
\text { /SCID-II })\end{array}$ & $\begin{array}{l}10 y \\
(64)\end{array}$ & $\begin{array}{l}\text { No controlled } \\
\text { treatment }\end{array}$ & $\begin{array}{l}64 \\
(41)\end{array}$ & $\begin{array}{l}26.9 \\
(92.7)\end{array}$ & 54 & $5(8)$ & $\begin{array}{l}55 \\
(\mathrm{DIB}-\mathrm{R}<6 \\
\text { /SCID-II) }\end{array}$ & $\begin{array}{l}\text { 17-HDRS/ } \\
\text { SASS }\end{array}$ \\
\hline $\begin{array}{l}\text { (11) Zeitler et al., } 2018 \\
\text { [13] }\end{array}$ & Germany & Moderate & $\begin{array}{l}\text { DSM-IV } \\
\text { (DIB-R } \\
\text { /SCID-II) }\end{array}$ & $\begin{array}{l}14.4 y \\
(35)\end{array}$ & $\begin{array}{l}\text { No controlled } \\
\text { treatment }\end{array}$ & $\begin{array}{l}167 \\
(58)\end{array}$ & $\begin{array}{l}29.2 \\
(100)\end{array}$ & - & - & $\begin{array}{l}81 \\
(\mathrm{IPDE}<5)\end{array}$ & - \\
\hline
\end{tabular}

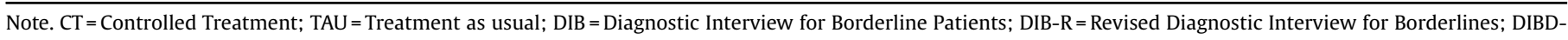

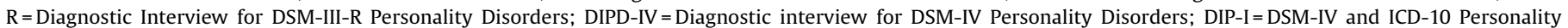

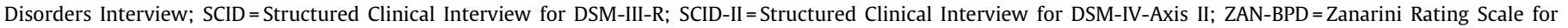

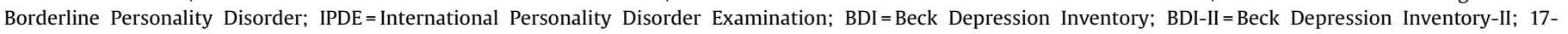

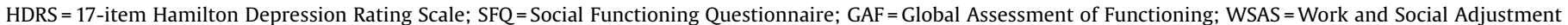

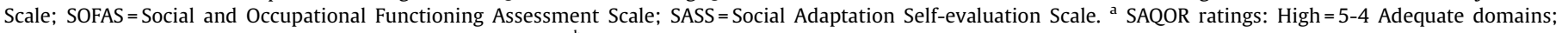

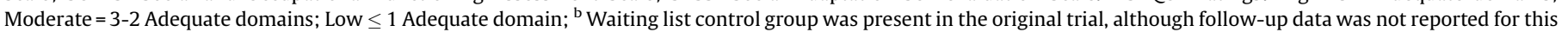

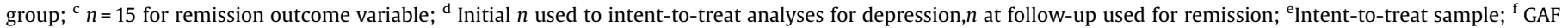
scores only reported at baseline in these studies.

Depressive symptoms were evaluated by the Beck Depression Inventory (BDI or BDI-II) and the 17-item Hamilton Rating Scale for Depression (HRSD-17). The Dysphoric Affect Scale (DAS) was used by the MSAD and was not considered comparable to the BDI/BDI-II and HRSD-17 due to the fact that this instrument identifies other dysphoric states apart from depression [51].

A variety of instruments were used as a measure of functioning, including (a) scales rated by clinicians: the Global Assessment of Functioning (GAF) and the Social and Occupational Functioning Assessment Scale (SOFAS), and (b) self-report questionnaires: the Social Functioning Questionnaire (SFQ), the Work and Social
Adjustment Scale (WSAS), and the Social Adjustment Scale-SelfReport (SASS). Results on GAF scores were partially reported by three studies and were not analysed $[9,20,49]$. Besides, the MSAD was also excluded due to reporting specific indexes of psychosocial functioning $[8,52]$.

\subsubsection{Treatment interventions}

Table 2 illustrates the controlled treatment conditions compared in the follow-up clinical trials included in the meta-analyses. Controlled treatments lasted for one to three years and were mainly conducted in outpatient settings, with the exception of two 


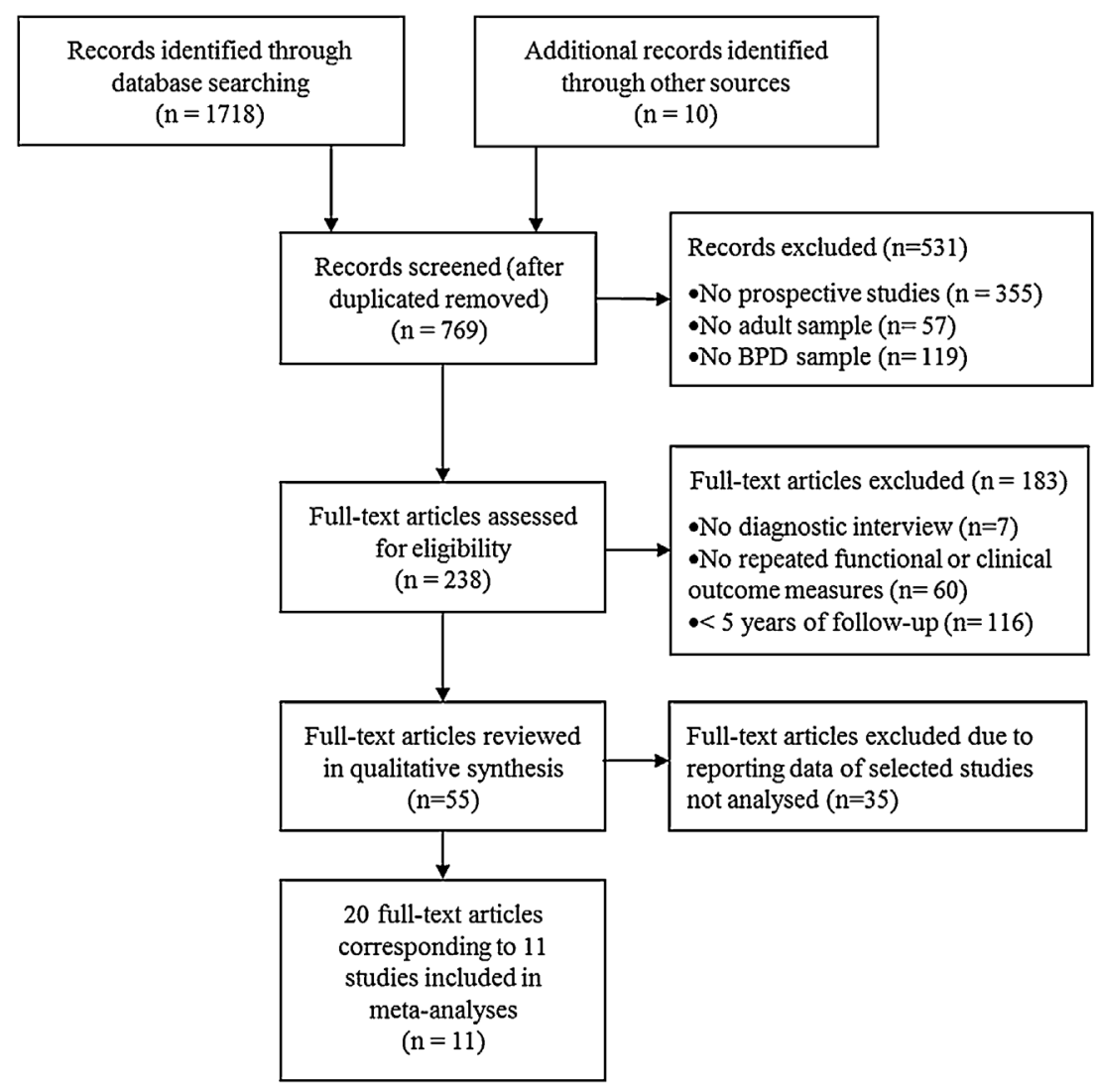

Fig. 1. Flow chart of selection process.

groups who received a combination of day hospital and outpatient treatment $[19,20]$. The hours of formal therapy fluctuated between 60 and over $600 \mathrm{~h}$, the latter reported by the MBT trial [20].

Among naturalistic studies, two studies followed up samples who initially participated in a clinical trial, although treatment subgroups were not individually studied at follow-up [10,13]. Besides, three naturalistic studies informed of treatment use during follow-up in general terms. The CLPS and MSAD studies reported similar percentages of subjects in BPD samples who participated in individual therapy during the early years of follow-up, which tended to decrease over time (range, $85 \%-64 \%$ and $96 \%-75 \%$, respectively) $[53,54]$. Álvarez-Tomás et al. also reported that $75 \%$ of subjects received individual therapy over the 10 -year period [10].

\subsection{Meta-analytic results}

There was no evidence for publication biases with respect to the four outcome measures studied in meta-analyses, according to the results of the Egger's regression test (Remission, $t=0.41, d f=10, p=$ .69; Completed suicide, $t=1.98, d f=8, p=.08$ ) and the trim and fill method (no study trimmed for depression and functioning).

\subsubsection{Remission}

Nine studies were analysed for remission, corresponding to twelve comparisons. The mean remission rate was 60\% (49-71, 95\% IC), although remission rates showed high heterogeneity among studies $\left(Q=57.9, p<.001 ; I^{2}=80.9 \%\right)$. The percentages of remission in each study are displayed in Table 1.

The results of the effects of moderators are summarised in Table 3. Groups with a younger mean age at baseline were more likely to show higher remission rates at follow-up $\left(Q_{M}=4.48, p=.03\right)$. Greater percentages of women in the samples were associated with higher remission rates in the long-term, although this tendency did not reach statistical significance $\left(Q_{M}=2.98, p=.08\right)$. Time of follow-up, percentage of comorbidity with mood disorders at baseline, and initial level of functioning did not demonstrate a significant influence on long-term remission. No significant differences in mean remission rates were observed between groups receiving and not receiving an initial controlled treatment, i.e., clinical trials vs naturalistic studies (61\% vs. 59\%). Among clinical trials, the mean remission rate in groups receiving a specialised therapy was higher than in those receiving TAU (70\% vs. 52\%), although this difference was not statistically significant. No relevant impact of the length of treatment or the hours of formal therapy received was found.

\subsubsection{Completed suicide}

Seven studies were considered for completed suicide, which represented ten comparisons. The mean suicide rate was $4 \%(2-5$, $95 \% \mathrm{CI})$; the values were homogeneous among studies $(Q=8.68, p=$ $\left..47 ; I^{2}=.0 \%\right)$. The percentages of completed suicide in the studies ranged from $0 \%$ to $8 \%$, as shown in Table 1 . Due to the low heterogeneity presented among studies, the effects of moderators were not studied on this outcome variable.

\subsubsection{Depression}

Four studies were studied for depression, comprising six comparisons. Meta-analytic results showed a significant reduction in depressive symptoms at follow-up, indicated by a medium mean effect size $(g=-0.70,[-1.04,-0.36] 95 \% \mathrm{CI}, p<.001)$. However, there was high heterogeneity among the studies $(Q=23.40, p<.001$; $I^{2}=78.6 \%$ ).

The percentage of comorbidity with mood disorders at baseline was not studied as a moderator on depression because there were no data from at least three studies. No relevant impact of 
Table 2

Description of controlled treatment conditions in follow-up clinical studies.

\begin{tabular}{|c|c|c|c|c|}
\hline Study & Type of Treatment & $\begin{array}{l}\text { Treatment } \\
\text { Setting }\end{array}$ & Description of Treatment conditions & $\begin{array}{l}\text { Hours therapy } \\
\text { (Months) }\end{array}$ \\
\hline $\begin{array}{l}\text { (1) Stevenson \& Meares, } 1992 \\
\text { Stevenson et al., } 2005[23,41]\end{array}$ & $\begin{array}{l}\text { Conversational Therapy } \\
\text { (ST) }\end{array}$ & Outpatient & $\begin{array}{l}2 \text { sessions/week, } 1 \text { h session duration. Manualised Psychodynamic- } \\
\text { Interpersonal Psychotherapy. } \\
\text { Optional inpatient stays when in crisis. Optional medication use. } \\
\text { Therapists: } 17 \text { psychiatrists, } 2 \text { psychiatric nurses, } 1 \text { psychologist. } \\
\text { Weekly supervision by audiotapes of sessions }\end{array}$ & $\begin{array}{l}96 \\
(12)\end{array}$ \\
\hline \multirow[t]{2}{*}{$\begin{array}{l}\text { (2) Bateman \& Fonagy, 1999, 2001, } \\
2008[20,42,43]\end{array}$} & $\begin{array}{l}\text { Mentalisation Based } \\
\text { Therapy } \\
\text { (ST) }\end{array}$ & $\begin{array}{l}\text { Day } \\
\text { Hospital } \\
\text { Outpatient }\end{array}$ & $\begin{array}{l}\text { DH: } 18 \text { months, } 1 \text { session/week psychoanalytic IT, } 3 \text { sessions/week } \\
\text { analytic GT ( } 1 \text { h each), } 1 / \text { week psychodrama GT }(1 \mathrm{~h}), 1 / \text { week } \\
\text { community meeting GT }(1 \mathrm{~h}) \text {. Average length }=1.45 \mathrm{y}, 62 \% \text { attendance at } \\
\text { psychotherapy sessions. } 1 / \text { month case management IT. Medication and } \\
1 / \text { month psychiatric consultations. } \\
\text { OT: } 18 \text { months } 2 \text { sessions/week analytic GT ( } 180 \text { hours over } 18 \text { months). } \\
75 \% \text { attendance at group therapy sessions. Medication and every } 3 \\
\text { months psychiatric consultations. Optional inpatient stays when in } \\
\text { crisis. } \\
\text { Manualised psychotherapy. Therapists: nurses. } 2 / \text { week Supervision. }\end{array}$ & $\begin{array}{l}612 \\
(36)\end{array}$ \\
\hline & TAU & Outpatient & $\begin{array}{l}18 \text { months: No formal psychotherapy. } 2 / \text { month individual community } \\
\text { support by mental health nurses, } 100 \% \text { attendance. Medication and } 2 / \\
\text { month psychiatric consultations on average. Optional psychiatric DH } \\
\text { (admission rate }=72 \% \text {, average length stay }=6 \text { months). Optional } \\
\text { inpatient stays when in crisis. } \\
18 \text { months: Medication and psychiatric consultations, community } \\
\text { support IT. Optional inpatient stays or psychiatric DH when in crisis. } \\
\text { Psychotherapy but not MBT IT when recommended. }\end{array}$ & $\begin{array}{l}- \\
(36)\end{array}$ \\
\hline $\begin{array}{l}\text { (3) Davidson et al., } 2010 \\
\text { Davidson, Norrie et al., } 2006 \\
\text { Palmer et al., } 2006\end{array}$ & $\begin{array}{l}\text { CBT-PD (ST) } \\
\text { TAU }\end{array}$ & Outpatient & $\begin{array}{l}\text { CBT-PD: } 12 \text { months, } 30 \text { IT sessions, } 1 \text { h duration. CBT for Cluster B PDs. } \\
\text { Manualised Psychotherapy. Trained therapists and weekly supervision. } \\
\text { TAU: Idem as comparison group. }\end{array}$ & $\begin{array}{l}16 \\
(12)\end{array}$ \\
\hline$[21,45,46]$ & TAU & Outpatient & $\begin{array}{l}\text { Minimum Treatment: OT General practitioner care }+ \text { Community } \\
\text { mental health teams (CMH, 90\% total sample). Optional psychological } \\
\text { intervention when in crisis. Optional Accident and Emergency (A\&E) } \\
\text { visits when self-harm episodes ( } 50 \% \text { total sample) and inpatient stays } \\
\text { when in crisis. Optional occupational and social attendance. }\end{array}$ & - \\
\hline \multirow[t]{2}{*}{$\begin{array}{l}\text { (4) Antonsen et al., } 2017 \\
\text { Arnevik et al., } 2010[19,47]\end{array}$} & $\begin{array}{l}\text { Combination Programme } \\
\text { (ST) }\end{array}$ & $\begin{array}{l}\text { Day } \\
\text { Hospital } \\
\text { Outpatient }\end{array}$ & $\begin{array}{l}\text { DH: } 18 \text { weeks, } 3-4 \text { days/week, psychodynamic GT, schema focused } \\
\text { cognitive GT, anxiety cognitive behavioural GT } \\
\text { OT combined psychotherapy: max } 4 \text { y, weekly } 1.5 \text {-h sessions GT, max } \\
2.5 y \text {, weekly IT. Written manual. Relational psychotherapy, group } \\
\text { analysis and self-psychology. Optional psychopharmacological } \\
\text { consultations by psychiatrist. }\end{array}$ & $\begin{array}{l}117,5 \\
(28)\end{array}$ \\
\hline & $\begin{array}{l}\text { OIP } \\
(\mathrm{TAU})\end{array}$ & Outpatient & $\begin{array}{l}\text { No manual. No specific psychotherapeutic model, duration or intensity } \\
\text { of treatment. } \\
\text { Optional psychopharmacological consultations by psychiatrist. }\end{array}$ & $\begin{array}{l}60 \\
(24)\end{array}$ \\
\hline \multirow[t]{3}{*}{ (5) Sahin et al., 2017 [22] } & $\begin{array}{l}\text { Object-relational } \\
\text { Psychotherapy (ST 1) }\end{array}$ & Outpatient & $\begin{array}{l}2 \text { sessions/week IT. Manualised psychotherapy. Trained therapists and } \\
\text { supervision. } \\
\text { Continuity of treatment after experimental phase was optional. }\end{array}$ & $\begin{array}{l}96 \\
(12)\end{array}$ \\
\hline & $\begin{array}{l}\text { Dialectical Behaviour } \\
\text { Therapy (ST 2) }\end{array}$ & Outpatient & $\begin{array}{l}1 \text { session/week IT, weekly } 2 \mathrm{~h} \text { sessions GT. Optional phone calls with } \\
\text { therapists between sessions. Manualised psychotherapy. Trained } \\
\text { therapists and supervision. } \\
\text { Continuity of treatment after experimental phase was optional. }\end{array}$ & $\begin{array}{l}144 \\
(12)\end{array}$ \\
\hline & TAU & Outpatient & Usual treatment in psychiatric units & $\begin{array}{l}- \\
(12)\end{array}$ \\
\hline
\end{tabular}

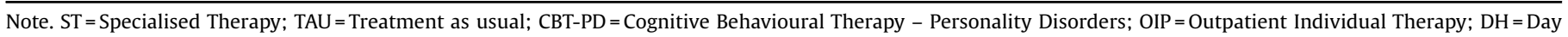

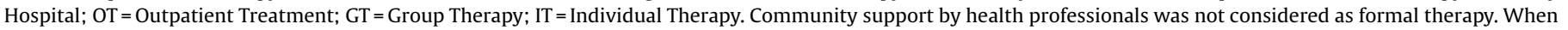
duration of sessions was not informed, it was computed $1 \mathrm{~h}$ per session. Real over planned data on use of therapy was preferred for calculations.

moderators on depression was found, except for the length of treatment. Longer treatments were associated with increased improvement in depression over time, although this trend did not reach statistical significance $\left(Q_{M}=3.01, p=.08\right)$.

\subsubsection{Functioning}

Five studies were synthesised for functioning, which represented nine comparisons. A significant improvement in long-term functioning was also found, with a medium mean effect size $(g=$ $0.66,[0.43,0.89] 95 \%$ IC, $p<.001)$. However, there was high heterogeneity among studies $\left(Q=25.54, p=.001 ; I^{2}=68.7 \%\right)$.

Groups with a higher percentage of women were more likely to present lower improvement in functioning at follow-up $\left(Q_{M}=7.24, p=\right.$ .007); age at baseline, time of follow-up, and initial level of functioning showed no relevant impact on long-term functioning. The initial rate of comorbidity with mood disorders was also not studied for functioning because there were no data from at least three studies. Differences in functional improvement between groups from naturalistic studies and those from clinical trials receiving a controlled treatment were not significant, although the latter showed a greater estimated effect size ( $g=0.43$ vs. $g=0.74, p=$ .11). In clinical trials, receiving specialised therapies or TAU did not show a relevant impact on functional change. There was a nonsignificant relationship between longer treatments and higher functional improvement $\left(Q_{M}=3.16, p=.07\right)$, whereas the hours of formal therapy received did not have a relevant influence on functioning.

\section{Discussion}

This meta-analytic study synthesised current findings from prospective research on the long-term course of BPD in adulthood. 
Table 3

Results of the effects of moderators on remission, depression, and functioning.

\begin{tabular}{|c|c|c|c|c|c|c|c|c|c|c|c|c|c|c|c|}
\hline \multirow{3}{*}{$\begin{array}{l}\text { Moderators } \\
\text { Mean Age }\end{array}$} & \multicolumn{5}{|c|}{ Remission } & \multicolumn{5}{|c|}{ Depression } & \multicolumn{5}{|c|}{ Functioning } \\
\hline & \multicolumn{2}{|c|}{ Estimate $[95 \% \mathrm{CI}]$} & \multirow{2}{*}{$\frac{Q_{M}}{4.48}$} & \multirow{2}{*}{$\frac{(d f)}{(1)}$} & \multirow{2}{*}{$\frac{p}{.03}$} & \multicolumn{2}{|c|}{ Estimate $[95 \% \mathrm{CI}]$} & \multirow{2}{*}{$\frac{Q_{M}}{2.72}$} & \multirow{2}{*}{$\frac{(d f)}{(1)}$} & \multirow{2}{*}{$\frac{p}{.10}$} & \multicolumn{2}{|c|}{ Estimate $[95 \% \mathrm{CI}]$} & \multirow{2}{*}{$\frac{Q_{M}}{0.41}$} & \multirow{2}{*}{$\frac{(d f)}{(1)}$} & \multirow{2}{*}{$\frac{p}{.52}$} \\
\hline & -0.13 & {$[-0.25,-0.01]$} & & & & 0.05 & {$[-0.01,0.12]$} & & & & 0.02 & {$[-0.04,0.08]$} & & & \\
\hline \% Women & 0.03 & {$[-0.00,0.06]$} & 2.98 & (1) & .08 & 0.01 & {$[-0.07,0.10]$} & 0.13 & (1) & .72 & -0.03 & {$[-0.05,-0.01]$} & 7.24 & (1) & .01 \\
\hline Time of Follow-up & 0.11 & {$[-0.08,0.30]$} & 1.23 & (1) & .27 & -0.07 & {$[-0.25,0.11]$} & 0.52 & (1) & .47 & -0.09 & {$[-0.23,0.05]$} & 1.68 & (1) & .19 \\
\hline \% Mood Disorders & 0.01 & {$[-0.07,0.09]$} & 0.04 & (1) & .84 & - & - & - & - & - & - & - & - & - & - \\
\hline Mean Initial Level of Functioning (z score) & 0.61 & {$[-0.32,1.54]$} & 1.67 & (1) & .20 & 0.18 & {$[-0.28,0.65]$} & 0.60 & (1) & .44 & -0.00 & {$[-0.38,0.37]$} & 0.00 & (1) & .98 \\
\hline Length of Treatment & 0.02 & {$[-0.06,0.12]$} & 0.33 & (1) & .57 & 0.04 & {$[-0.01,0.08]$} & 3.01 & (1) & .08 & 0.03 & {$[-0.00,0.07]$} & 3.16 & (1) & .07 \\
\hline \multirow[t]{2}{*}{ Hours of Formal Therapy } & 0.00 & {$[-0.00,0.01]$} & 1.02 & (1) & .31 & 0.00 & {$[-0.01,0.01]$} & 0.15 & (1) & .70 & -0.00 & {$[-0.01,0.00]$} & 1.13 & (1) & .29 \\
\hline & \multicolumn{2}{|c|}{ Rate $[95 \% \mathrm{CI}]$} & $Q_{B}$ & $(d f)$ & $p$ & \multicolumn{2}{|c|}{$g[95 \% \mathrm{CI}]$} & $Q_{B}$ & $(d f)$ & $p$ & \multicolumn{2}{|c|}{$g[95 \% \mathrm{CI}]$} & $Q_{B}$ & $(d f)$ & $p$ \\
\hline Presence of Controlled Treatment & & & 0.03 & (1) & .85 & & & 0.22 & (1) & .64 & & & 2.50 & (1) & .11 \\
\hline No Controlled Treatment & $59 \%$ & {$[.42, .74]$} & & & & -0.58 & {$[-1.21,-0.06]$} & & & & 0.43 & {$[0.17,0.69]$} & & & \\
\hline Controlled Treatment & $61 \%$ & {$[.45, .76]$} & & & & -0.77 & {$[-1.23,-0.30]$} & & & & 0.74 & {$[0.45,1.03]$} & & & \\
\hline Type of Controlled Treatment & & & 0.57 & (1) & .45 & & & 0.52 & (1) & .47 & & & 0.08 & (1) & .77 \\
\hline Specialised Therapy & $70 \%$ & {$[.41, .89]$} & & & & -0.95 & {$[-1.68,-0.23]$} & & & & 0.79 & {$[0.26,1.31]$} & & & \\
\hline TAU & $52 \%$ & {$[.20, .83]$} & & & & -0.58 & {$[-1.30,0.15]$} & & & & 0.70 & {$[0.47,0.93]$} & & & \\
\hline
\end{tabular}

In terms of mean remission rates, it is estimated that between $50 \%$ and $70 \%$ of patients diagnosed with BPD may achieve symptomatic remission at some point between five and fifteen years of followup; this finding is consistent with former literature indicating that BPD diagnosis leans towards increasing proportions of remission over time. The Montreal study retrospectively reported that $75 \%$ of patients with BPD achieved remission at fifteen years and over $90 \%$ did so at 27 years of follow-up [2]. Both the MSAD and CLPS studies also provided evidence for an enduring symptomatic remission of $\mathrm{BPD}$, despite reporting cumulative rates of remission [6]. To our knowledge, our study is the first meta-analysis reporting estimated remission rates prospectively assessed at a specific point, which may be useful in comparing the long-term effects of treatments for BPD patients over the course of the disorder.

Notably, depressive symptomatology and functioning in patients with BPD also lean towards improvement in the longterm. Findings are consistent with those reported by the CLPS and MSAD studies, indicating that the psychosocial functioning of BPD subjects may vary among individuals, but show a significant improvement over time as a group [6]. However, our results do not allow one to conclude whether individuals with BPD can reach normative functional adjustment in the long-term, although previous research indicates that a relevant proportion may suffer from persistent impairments over time $[8,9,10,13,52]$. In terms of depressive symptoms, the MSAD study also reported a significant decrease in dysphoric states in the long-term, which was more pronounced in recovered patients [51]. Despite this favourable outcome in clinical and functional realms, we found that the rate of completed suicide in BPD subjects might be expected to be between $2 \%$ and $5 \%$ during the second half of the decade of followup; percentages were lower than those based on previous followback research at fifteen and 27 years, which were between $8 \%$ and $10 \%$. This fact is consistent with the hypothesis that completions are more likely to occur later in the course of the illness [1,2].

We further investigated potential moderators for those outcomes which presented high heterogeneity in the meta-analyses. Regarding patients' age at baseline, we found that a diagnosis of BPD at a younger age was associated with higher percentages of remission in the long-term, whereas this moderator did not influence changes in depression and functioning. This is congruent with previous evidence indicating that BPD symptom severity may decline from adolescence to mid-adulthood, particularly in terms of the externalising manifestations of the disorder (i.e., impulsivity and suicidal behaviours) $[55,56,57,58]$. Younger age has also been identified as a predictor of shorter time to remission [14]. In contrast, the CLPS informed of similar rates of improvement in BPD symptoms in younger and older subjects over six years of followup [15]. This discrepancy in longitudinal findings suggests that age-related divergences might also be influenced by the duration of the illness, which is expected to be longer the older one becomes but with variations among subjects. Similarly, it would be useful to consider the age of onset and the duration of illness as specific moderators when studying the course of the disorder. In terms of functioning, cross-sectional research has reported, contrary to our findings, greater functional impairments in older age, when it is more likely to present worse physical health, poorer quality of life, and greater social assistance utilisation [56,57,58]. Nevertheless, this decline in functioning is more dramatically suffered in advanced age, whereas subjects comprising the study samples in our analysis were mostly in their $20 \mathrm{~s}$ and $30 \mathrm{~s}$ at baseline. Consistent with this hypothesis, the CLPS study reported that older participants only showed a higher decline in functioning midway through the follow-up [15].

A striking finding was that female gender was associated with a lower improvement in functioning, whereas there were no significant differences in gender with regard to changes in BPD diagnosis and depression in the long-term. These results are consistent with those informed by the MSAD, which did not report a significant impact of gender on time to remission over ten years [14]. Unfortunately, there is scarce evidence on gender differences regarding the functional course of BPD. In this respect, our findings indicate that the long-term functional recovery in women with BPD might be hampered by other causes apart from the persistence of the disorder. A possible explanation is that psychopathological divergences between genders in symptom severity and comorbidity might deeply interfere with the long-term functional outcome of BPD women, in spite of showing similar trends of clinical improvement over time. In this line, there is evidence from crosssectional research on gender differences that female BPD subjects present greater percentages of lifetime Axis I comorbidity and higher severity in general psychopathology, particularly in areas related to internalising patterns such as anxiety, depression, and somatisation [59,60]. Additionally, BPD women are more likely to have a history of childhood sexual abuse and experience episodes of physical and sexual aggression during adulthood, which is related to higher psychosocial impairment [59,61]. On the other hand, sociological studies have noted that gender roles determine different strains and benefits of normative social adaptation for men and women, which might also contribute to hindering psychosocial adjustment in female BPD subjects; for example, caregiving roles for women in the general population are associated with greater psychological distress and increased 
reductions in the protective benefits of employment for mental health, reporting higher rates of exposure to stressful life events involving significant others [62,63].

Receiving an initial controlled treatment does not seem to increase the amount of clinical improvement in the long-term compared to the course of the disorder described in naturalistic studies. Besides, both specialised therapies and TAU seem to similarly improve the long-term clinical outcome, despite observing non-significant differences in mean remission rates between those groups. This contrasts with previous research indicating a higher efficacy of specialised therapies for BPD in the short-term $[64,65]$, which suggests that the differential effects of psychotherapeutic interventions might be diluted by the diverse mechanisms of change over the lifespan. On the other hand, the long-term functioning of BPD patients appears to be enhanced by any kind of controlled treatment, particularly those that are implemented over a longer period of time, although these trends did not reach statistical significance in our analyses. This might indicate that common components of psychotherapies are key factors in promoting changes in psychosocial functioning in the long-term [66]. One aspect to note is that the methodological limitations of the selected studies may affect these results on treatment moderators. First, other confounding factors are likely to interfere with treatment efficacy in long-lasting post-treatment follow-ups, including the likelihood of receiving further treatment after the experimental phase. Secondly, most BPD subjects in naturalistic studies might also have received some form of uncontrolled treatment during the follow-up period $[2,64]$.

The length of follow-up was not relevant to explain the variations in the outcomes, suggesting a low pace of change in the long-term. This differs from the early years of illness when more dramatic shifts in BPD symptoms may occur $[1,14]$. Additionally, no significant effects of the initial level of functioning and the percentage of comorbid mood disorders were found; this contrasts with findings from the CLPS indicating that a comorbid major depressive disorder is associated with a delayed time to remission from BPD, likely due to differences in the operationalisation of remission in this study [16].

There are strengths and limitations of this meta-analysis to consider. We included follow-along studies conducted in a variety of clinical settings and countries, which increases the generalisation of the findings. We also analysed the impact of several factors on the heterogeneity of the outcomes; however, the majority of selected studies reported results at follow-up lasting between five and ten years, largely restricting the scope of our findings to this time period. In general, the scarcity of selected studies and the small size of the BPD samples in most of them reduced the statistical power of the analyses and therefore may have affected the study of the effects of moderators. Additionally, the lack of data or the variability of measures used among studies restricted the analysis of other potential moderators and outcomes. Finally, we studied correlational relationships between moderators and longterm outcomes and, therefore, we cannot establish causal links based on our findings.

\section{Conclusion}

Our findings confirm a pattern of clinical and functional improvement over time in patients diagnosed with BPD. It is tempting to think about the beneficial effects of psychotherapeutic interventions, specialised or not, on the functional long-term course, but further research on the long-term effects of psychotherapies is required to reach consistent conclusions in this regard. Moreover, longitudinal studies in untreated samples might also enrich our knowledge of the natural course of BPD and the study of treatment efficacy. In general, the consistent use of clinical and functional measures for BPD would facilitate meta-analytic research in this field.

Our results lead to the conclusion that BPD diagnosis at a younger age corresponds to a better clinical prognosis in the long-term, whereas similar rates of functional improvement are achieved by age over time. This justifies direct efforts towards the early detection of $\mathrm{BPD}$, allowing the implementation of effective treatments during patients' youth to reduce the adverse effects of the disorder during this critical life stage. In addition, functional improvement in the long-term is hampered in women with BPD; accordingly, it is essential to incorporate a gender perspective to address psychosocial interventions for these patients, particularly due to the higher percentage of women among treatment-seekers with BPD diagnosis. Moreover, the use of study designs that allow gender comparisons would be recommended.

\section{Financial support}

This research did not receive any specific grant from funding agencies in the public, commercial, or not-for-profit sectors.

\section{Disclosure of interest}

The authors declare that they have no competing interests.

\section{References}

[1] Biskin RS. The lifetime course of borderline personality disorder. Can ] Psychiatry 2015;60(7):303-8.

[2] Paris J. Implications of long-term outcome research for the management of patients with borderline personality disorder. Harv Rev Psychiatry 2002;10 (6):315-23.

[3] Ng FYY, Bourke ME, Grenyer BFS. Recovery from borderline personality disorder: a systematic review of the perspectives of consumers, clinicians, family and carers. PloS One 2016;11(8):1, doi:http://dx.doi.org/10.1371/ journal.pone.0160515.

[4] Skodol AE, Gunderson JG, Shea MT, McGlashan TH, Morey LC, Sanislow CA et al. The collaborative longitudinal personality disorders study (CLPS): overview and implications. J Pers Disord 2005;19(5):487-504, doi:http://dx. doi.org/10.1521/pedi.2005.19.5.487.

[5] Zanarini MC, Frankenburg FR, Hennen J, Reich DB, Silk KR. The McLean Study of Adult Development (MSAD): overview and implications of the first six years of prospective follow-up. J Pers Disord 2005;19(5):505-23.

[6] Zanarini MC. Diagnostic specificity and long-term prospective course of borderline personality disorder. Psychiatr Ann 2012;42(2):53-8, doi:http://dx. doi.org/10.3928/00485713-20120124-05.

[7] Zanarini MC, Frankenburg FR, Reich DB, Fitzmaurice G. Attainment and stability of sustained symptomatic remission and recovery among patients with borderline personality disorder and axis II comparison subjects: a 16year prospective follow-up study. Am J Psychiatry 2012;169:476-83.

[8] Zanarini MC, Frankenburg FR, Hennen J, Reich DB, Silk KR. Psychosocial functioning of borderline patients and axis II comparison subjects followed prospectively for six years. J Pers Disord 2005;19(1):19-29.

[9] Gunderson JG, Stout RL, McGlashan TH, Shea MT, Morey LC, Grilo CM, et al. Tenyear course of borderline personality disorder: psychopathology and function from the Collaborative Longitudinal Personality Disorders Study. Arch Gen Psychiatry 2011;68(8):827-37.

[10] Alvarez-Tomás I, Soler J, Bados A, Martín-Blanco A, Elices M, Carmona C, et al. Long-term course of borderline personality disorder: a prospective 10-year follow-up study. J Pers Disord 2017;31(5):590-605, doi:http://dx.doi.org/ 10.1521/pedi_2016_30_269.

[11] Links PS, Heslegrave R, Van Reekum R. Prospective follow-up study of borderline personality disorder: prognosis, prediction of outcome, and Axis II comorbidity. Can J Psychiatry Rev Can Psychiatr 1998;43(3):265-70.

[12] Riihimäki K, Vuorilehto M, Isometsä E. Borderline personality disorder among primary care depressive patients: a five-year study. J Affect Disord 2014;155:303-6, doi:http://dx.doi.org/10.1016/j.jad.2013.10.050.

[13] Zeitler M, Bohus M, Kleindienst N, Knies R, Ostermann M, Schmahl C, et al. How to assess recovery in borderline personality disorder: psychosocial functioning and satisfaction with life in a sample of former DBT study patients. J Personal Disord 2018;04(September):1-19.

[14] Zanarini MC, Frankenburg FR, Hennen J, Reich DB, Silk KR. Prediction of the 10 year course of borderline personality disorder. Am J Psychiatry 2006;163 (5):827-32.

[15] Shea MT, Edelen MO, Pinto A, Yen S, Gunderson JG, Skodol AE, et al. Improvement in borderline personality disorder in relationship to age. Acta Psychiatr Scand 2009;119:143-8. 
[16] Gunderson JG, Stout RL, Shea MT, Grilo CM, Markowitz JC, Morey LC, et al. Interactions of borderline personality disorder and mood disorders over 10 years. J Clin Psychiatry 2014;75(8):829-34.

[17] Soloff PH, Chiappetta L. Prospective predictors of suicidal behavior in borderline personality disorder at 6-year follow-up. Am J Psychiatry 2012;169(5):484-90, doi:http://dx.doi.org/10.1176/appi.ajp.2011.11091378.

[18] Wedig MM, Frankenburg FR, Bradford Reich D, Fitzmaurice G, Zanarini MC. Predictors of suicide threats in patients with borderline personality disorder over 16 years of prospective follow-up. Psychiatry Res 2013;208 (3):252-6.

[19] Antonsen BT, Kvarstein EH, Urnes ø, et al. Favourable outcome of long-term combined psychotherapy for patients with borderline personality disorder: six-year follow-up of a randomized study. Psychother Res 2017;27(1):51-63.

[20] Bateman A, Fonagy P. 8-year follow-up of patients treated for borderline personality disorder: mentalization-based treatment versus treatment as usual. Am J Psychiatry 2008;165(5):631-8.

[21] Davidson KM, Tyrer P, Norrie J, Palmer SJ, Tyrer H. Cognitive therapy v. Usual treatment for borderline personality disorder: prospective 6-year follow-up. Br J Psychiatry 2010;197:456-62.

[22] Sahin Z, Vinnars B, Gorman BS, Wilczek A, Åsberg M, Barber JP. Clinical severity as a moderator of outcome in psychodynamic and dialectical behavior therapies for borderline personality disorder. Personal Disord 2017;14 (December), doi:http://dx.doi.org/10.1037/per0000276.

[23] Stevenson J, Meares R, D'Angelo R. Five-year outcome of outpatient psychotherapy with borderline patients. Psychol Med 2005:35(1):79-87.

[24] Liberati A, Altman DG, Tetzlaff J, Mulrow C, Gøtzsche PC, et al. The PRISMA statement for reporting systematic reviews and meta-analyses of studies that evaluate health care interventions: explanation and elaboration. PLoS Med 2009;6(7)e1000100, doi:http://dx.doi.org/10.1371/journal. pmed.1000100.

[25] Bobes J, González MP, Bascarán MT, Corominas A, Adan A, Sánchez J, et al. Validación de la versión española de la Escala de Adaptación Social en pacientes depresivos [Validation of the Spanish version of the Social Adaptation Scale in depressive patients]. Actas Españolas Psiquiatr 1999;27 (2):71-80.

[26] Greenberg GA, Rosenheck RA. Using the GAF as a national mental health outcome measure in the Department of Veterans Affairs. Psychiatr Serv 2005;56(4):420-6.

[27] Pedersen G, Kvarstein EH, Wilberg T. The Work and Social Adjustment Scale: Psychometric properties and validity among males and females, and outpatients with and without personality disorders. Personal Ment Health 2017;11(4):215-28, doi:http://dx.doi.org/10.1002/pmh.1382.

[28] Suominen K, Salminen E, Lähteenmäki S, Tupala T, Isometsä E. Validity and reliability of the Finnish version of the Functioning Assessment Short Test (FAST) in bipolar disorder. Int J Bipolar Disord 2015;3(10), doi:http://dx.doi. org/10.1186/s40345-015-0025-1.

[29] Tungström S, Söderberg P, Armelius B-A. Relationship between the Global Assessment of Functioning and other DSM axes in routine clinical work. Psychiatr Serv 2005;56(4):439-43.

[30] Tyrer P, Nur U, Crawford M, Karlsen S, MacLean C, Rao B, et al. The social functioning questionnaire: a rapid and robust measure of perceived functioning. Int J Soc Psychiatry 2005;51(3):265-75, doi:http://dx.doi.org $10.1177 / 0020764005057391$.

[31] Ross LE, Grigoriadis S, Mamisashvili L, Koren G, Steiner M, Dennis C-L, et al. Quality assessment of observational studies in psychiatry: an example from perinatal psychiatric research. Int J Methods Psychiatr Res 2011;20(4):224-34, doi:http://dx.doi.org/10.1002/mpr.356.

[32] Betancourt TS, Borisova I, Williams TP, Meyers-Ohki SE, Rubin-Smith JE, Annan J, et al. Research Review: psychosocial adjustment and mental health in forme child soldiers - a systematic review of the literature and recommendations for future research. J Child Psychol Psychiatry 2013;54(1):17-36.

[33] Borenstein M, Hedges LV, Higgins JP, Rothstein HR. Introduction to metaanalysis. West Sussex, UK: John Wiley \& Sons; 2011.

[34] Egger M, Smith GD, Schneider M, Minder C. Bias in meta-analysis detected by a simple, graphical test. BMJ 1997;315(7109):629-34.

[35] Duval S, Tweedie R. Trim and fill: a simple funnel-plot-based method of testing and adjusting for publication bias in meta-analysis. Biometrics 2000;56 (2):455-63.

[36] Sterne JAC, Sutton AJ, Ioannidis JPA, Terrin N, Jones DR, Lau J, et al. Recommendations for examining and interpreting funnel plot asymmetry in meta-analyses of randomised controlled trials. BMJ 2011;22(July) 343:1..

[37] Hedges LV, Olkin I. Statistical methods for meta-analysis. Orlando: Academic Press; 1985.

[38] Higgins JPT, Thompson SG. Quantifying heterogeneity in a meta-analysis. Stat Med 2002;21(11):1539-58.

[39] Viechtbauer W. Bias and efficiency of meta-analytic variance estimators in the random-effects model. J Educ Behav Stat 2005;30:261-93.

[40] Borenstein M, Rothstein H. Comprehensive Meta-Analysis: A Computer Program For Research Synthesis. New Jersey: Biostat.; 1999.

[41] Stevenson J, Meares R. An outcome study of psychotherapy for patients with borderline personality disorder. Am J Psychiatry 1992;149(3):358-62.

[42] Bateman A, Fonagy P. Effectiveness of partial hospitalization in the treatment of borderline personality disorder: a randomized controlled trial. Am J Psychiatry 1999;156(10):1563-9.
[43] Bateman A, Fonagy P. Treatment of borderline personality disorder with psychoanalytically oriented partial hospitalization: an 18- month follow-up. Am J Psychiatry 2001;158(1):36-42.

[44] Davidson K, Tyrer P, Gumley A, Tata P, Norrie J, Palmer S, et al. A randomized controlled trial of cognitive behavior therapy for borderline personality disorder: rationale for trial, method and description of sample. J Pers Disord 2006;20(5):431-49.

[45] Davidson K, Norrie J, Tyrer P, Gumley A, Tata P, Murray H, et al. The effectiveness of cognitive behavior therapy for borderline personality disorder: results from the borderline personality disorder study of cognitive therapy (BOSCOT) trial. J Pers Disord 2006;20(5):450-65.

[46] Palmer S, Davidson K, Tyrer P, Gumley A, Tata P, Norrie J, et al. The costeffectiveness of cognitive behavior therapy for borderline personality disorder: results from the BOSCOT trial. J Pers Disord 2006;20(5):466-80.

[47] Arnevik E, Wilberg T, Urnes $\varnothing$, Johansen M, Monsen JT, Karterud S. Psychotherapy for personality disorders: 18 months' follow-up of the ullevål personality project. J Pers Disord 2010;24(2):188-203, doi:http://dx. doi.org/10.1521/pedi.2010.24.2.188

[48] Links PS, Heslegrave R, Mitton JE, van Reekum R, Patrick J. Borderline psychopathology and recurrences of clinical disorders. J Nerv Ment Dis 1995;183(9):582-6.

[49] Zanarini MC, Frankenburg FR, Hennen J, Silk KR. The longitudinal course of borderline psychopathology: 6-year prospective follow-up of the phenomenology of borderline personality disorder. Am J Psychiatry 2003;160 (2):274-83, doi:http://dx.doi.org/10.1521/ijct.2009.2.4.343.

[50] Skodol AE, Pagano ME, Bender DS, Shea MT, Gunderson JG, Yen S, et al. Stability of functional impairment in patients with schizotypal, borderline, avoidant, or obsessive-compulsive personality disorder over two years. Psychol Med 2005;35(3):443-51, doi:http://dx.doi.org/10.1017/S003329170400354X.

[51] Reed LI, Fitzmaurice G, Zanarini MC. The course of dysphoric affective and cognitive states in borderline personality disorder: a 10-year follow-up study. Psychiatry Res 2012;196(1):96-100, doi:http://dx.doi.org/10.1016/j. psychres.2011.08.026.

[52] Zanarini MC, Frankenburg FR, Reich DB, Fitzmaurice G. The 10-year course of psychosocial functioning among patients with borderline personality disorder and axis II comparison subjects. Acta Psychiatr Scand 2010;122(2):103-9.

[53] Bender DS, Skodol AE, Pagano M, Dyck I, Grilo C, Shea M, et al. Prospective assessment of treatment use by patients with personality disorders. Psychiatr Serv 2006:57(2):254-7.

[54] Zanarini MC, Frankenburg FR, Hennen J, Silk KR. Mental health service utilization by borderline personality disorder patients and Axis II comparison subjects followed prospectively for 6 years. J Clin Psychiatry 2004;65(1):28-36.

[55] Blum N, Franklin J, Hansel R, McCormick B, St.John D, Pfohl B, et al. Relationship of age to symptom severity, psychiatric comorbidity and health care utilization in persons with borderline personality disorder. Personal Ment Health 2008; 2:25-34, doi:http://dx.doi.org/10.1002/pmh.26.

[56] Frías Á, Palma C, Solves L, Martínez B, Salvador A. Differential symptomatology and functioning in borderline personality disorder across age groups. Psychiatry Res 2017;258:44-50, doi:http://dx.doi.org/10.1016/j. psychres.2017.09.081.

[57] Morgan TA, Chelminski I, Young D, Dalrymple K, Zimmerman M. Differences between older and younger adults with borderline personality disorder on clinical presentation and impairment. J Psychiatr Res 2013;47(10):1507-13, doi:http://dx.doi.org/10.1016/j.jpsychires.2013.06.009.

[58] Stepp SD, Pilkonis PA. Age-related differences in individual DSM criteria for borderline personality disorder. J Pers Disord 2008;22(4):427-32, doi:http:// dx.doi.org/10.1521/pedi.2008.22.4.427.

[59] Bayes A, Parker G. Borderline personality disorder in men: a literature review and illustrative case vignettes. Psychiatry Res 2017;257:197-202, doi:http:// dx.doi.org/10.1016/j.psychres.2017.07.047.

[60] Silberschmidt A, Lee S, Zanarini M, Schulz SC. Gender differences in borderline personality disorder: results from a multinational, clinical trial sample. J Pers Disord 2015;29(6):828-38, doi:http://dx.doi.org/10.1521/pedi_2014_28_175.

[61] de Aquino Ferreira LF, Queiroz Pereira FH, Neri Benevides AML. Aguiar Melo MC. Borderline personality disorder and sexual abuse: a systematic review. Psychiatry Res 2018;262:70-7, doi:http://dx.doi.org/10.1016/j. psychres.2018.01.043.

[62] Hatch SL, Dohrenwend BP. Distribution of traumatic and other stressful life events by race/ethnicity, gender, SES and age: a review of the research. Am J Community Psychol 2007;40(3-4):313-32.

[63] Depression Leupp K. Work and Family Roles, and the Gendered Life Course. J Health Soc Behav 2017;58(4):422-41, doi:http://dx.doi.org/10.1177/ 0022146517737309.

[64] Cristea IA, Gentili C, Cotet CD, Palomba D, Barbui C, Cuijpers P. Efficacy of psychotherapies for borderline personality disorder: a systematic review and meta-analysis. JAMA Psychiatry 2017;74(4):319-28, doi:http://dx.doi.org/ 10.1001/jamapsychiatry.2016.4287.

[65] Stoffers JM, Völlm BA, Rücker G, Timmer A, Huband N, Lieb K. Psychological therapies for people with borderline personality disorder. Cochrane Database Syst Rev 2012;8(1), doi:http://dx.doi.org/10.1002/14651858.CD005652.pub2.

[66] Sinnaeve R, van den Bosch LMC, van Steenbergen-Weijenburg KM. Change in interpersonal functioning during psychological interventions for borderline personality disorder-a systematic review of measures and efficacy. Personal Ment Health 2015;9(3):173-94, doi:http://dx.doi.org/10.1002/pmh.1296. 